\title{
Delay-induced self-organization dynamics in a prey-predator network with diffusion
}

\author{
Q.Hu · J.W.Shen
}

Received: date/Accepted: date

\begin{abstract}
Time delays can induce the loss of stability and degradation of performance. In this paper, the pattern dynamics of a prey-predator network with diffusion and delay are investigated, where the inhomogeneous distribution of species in space can be viewed as a random network, and delay can affect the stability of the network system. Our results show that time delay can induce the emergence of Hopf and Turing bifurcations, which are independent of the network, and the conditions of bifurcation are derived by linear stability analysis. Moreover, we find that the Turing pattern can be related to the network connection probability. The Turing instability region involving delay and network connection probability is obtained. Finally, the numerical simulation verifies our results.
\end{abstract}

Keywords Hopf bifurcation · Turing instability · prey-predator · delay · network diffusion

\section{Introduction}

Colorful patterns emerge from reaction-diffusion equations and can explain a wide variety of natural phenomena. Since Turing published his groundbreaking work [1], the Turing pattern has received increasing attention in biology, physics, chemicals, and other fields $[2,3,4,5]$. At present, it has been applied to more complex interdisciplinary subjects. Recently, many scientists have also found Turing patterns in prey-predator systems $[6,7,8]$. For the prey-predator

Q.Hu

School of Mathematics and Statistics, North China University of Water Resources and Electric Power, Zhengzhou 450046, China.

J.W. Shen

School of Mathematics and Statistics, North China University of Water Resources and

Electric Power, Zhengzhou 450046, China.

Corresponding E-mail: phdshen@126.com 
model, there are many variations in this model; for example, Aziz-Alaoui and Daher [9] proposed a modified predator-prey model, and Leslie [10] studied the boundedness and global stability of the model.

For a reaction-diffusion system, Turing [1] first discovered that diffusion could cause the system to be unstable under certain conditions and obtained Turing pattern phenomena. Then, many scholars began to study the pattern formations of the prey-predator model by using the reaction-diffusion equation $[11,12,13,14,15]$. Camara and Aziz-Alaoui [16] investigated a modified Holling-Tanner model and found Turing and Hopf bifurcations; they also derived the bifurcation conditions. Then, for a diffusive predator-prey model with the Holling-Tanner functional response, Shi et al. [17] investigated the stability and the existence of a positive solution.

By studying diffusion, Othmer and Scriven [18] proposed a general method for analyzing the instability of the reaction-diffusion model in small networks, and they also obtained the effect of network topology changes on the stability and pattern. Then, Jansen and Lloyd [19] extended the method to large random networks and gave the corresponding Turing patterns. Aly and Farkas [20] studied the effect of diffusion in a small network on the Turing pattern of the prey-predator system, where the migration of populations among patches causes diffusion. Subsequently, Turing patterns in complex organized networks were studied in $[21,22,23]$. For the FitzHugh-Nagumo model in a networkorganized system, Zheng and Shen [24] recently studied Turing instability, where the network structure and diffusion lead to Turing instability. Liu et al. [25] placed the prey-predator model into complex networks and found that the network topology and diffusion rate can affect the Turing pattern formation. Considering the inhomogeneous distribution of prey and predators in communities and the fact that communities in real life can be abstracted as a complex network structure, we can investigate a prey-predator diffusive system functional response in the random network.

Time delay is a universal phenomenon in biological systems [26,27,28]. For the prey-predator system, delay generally represents the gestation or maturity period of population reproduction; it can destroy the stability and lead to Hopf bifurcation as well as Turing bifurcation. Ruan and Sen [29,30] provided two methods to investigate the time delay. In [31], Yang and Zhang considered a diffusive prey-predator system with delay, and they obtained Turing instability and Hopf bifurcation caused by the delay. Recently, Chang et al. [32] investigated a predator-prey diffusive model in complex networks and found that delay can induce pattern dynamics. Wang et al. [33] found that the network structure hardly has an impact on the stability of this model with delay and that a large time delay can induce the thick-tailed phenomenon of evolution patterns.

However, there are few studies on reaction-diffusion systems with delays in diffusion networks. In this paper, for a prey-predator diffusion network with Holling type II and modified Leslie-Gower functional response, we will investigate the dynamical behaviors emerging from the time delay caused by predator gestation. For the random migration and inhomogeneous spatial distribution 
of prey and predators among different communities, we consider them through a complex network and derive the stability condition of the positive equilibrium by linear stability analysis in Section 2. Then, in Section 3, we use two methods to study Hopf and Turing bifurcations caused by delay and network diffusion. In Section 4, consistent results are obtained by numerical simulation. Finally, the results are summarized in Section 5.

\section{Stability analysis of the prey-predator diffusion network without delay}

The modified Leslie-Gower prey-predator diffusion network with a Holling type II functional response can be written as follows:

$$
\left\{\begin{array}{l}
\frac{\partial u}{\partial t}=u(1-u)-\frac{u v}{u+a}+d_{1} \nabla^{2} u=f(u, v)+d_{1} \nabla^{2} u \\
\frac{\partial v}{\partial t}=v\left(\delta-\beta \frac{v}{u+b}\right)+d_{2} \nabla^{2} v=g(u, v)+d_{2} \nabla^{2} v
\end{array}\right.
$$

where $\frac{v}{u+b}$ is the modified Leslie-Gower functional response.

Considering the inhomogeneous distribution of species and the effect of delay $\tau(\tau>0)$ caused by predator gestation, model (1) with delay in the random network is

$$
\left\{\begin{array}{l}
\frac{d u_{i}}{d t}=u_{i}\left(1-u_{i}\right)-\frac{u_{i} v_{i}(t-\tau)}{u_{i}+a}+d_{1} \sum_{j=1}^{N} L_{i j} u_{j}, \\
\frac{d v_{i}}{d t}=v_{i}\left(\delta-\beta \frac{v_{i}(t-\tau)}{u_{i}+b}\right)+d_{2} \sum_{j=1}^{N} L_{i j} v_{j},
\end{array}\right.
$$

where $i=1, \ldots, N, L_{i j}=A_{i j}-k_{i} \delta_{i j}$ is the network Laplacian matrix's element, where $k_{i}$ is the degree of node $i$ (we assume that $k_{i}$ is a decreasing sequence, that is, $k_{1} \geq k_{2} \geq \cdots \geq k_{N}$ ) and $A_{i j}$ is the adjacency matrix's element. $N$ nodes make up the random network, and the nodes are connected with the connection probability $p$ [24].

If $\tau=0$, the system (2) becomes:

$$
\left\{\begin{array}{l}
\frac{d u_{i}}{d t}=f\left(u_{i}, v_{i}\right)+d_{1} \sum_{j=1}^{N} L_{i j} u_{j} \\
\frac{d v_{i}}{d t}=g\left(u_{i}, v_{i}\right)+d_{2} \sum_{j=1}^{N} L_{i j} v_{j}
\end{array}\right.
$$

Clearly, the equilibrium points of system (3) are derived by the following equations:

$$
\left\{\begin{array}{l}
f\left(u_{i}, v_{i}\right)=u_{i}\left(1-u_{i}\right)-\frac{u_{i} v_{i}}{u_{i}+a}=0 \\
g\left(u_{i}, v_{i}\right)=v_{i}\left(\delta-\beta \frac{v_{i}}{u_{i}+b}\right)=0
\end{array}\right.
$$

Then, when $a-\frac{\delta}{\beta} b>0$, we obtain only one positive equilibrium point $\mathrm{E}$ $\left(u^{*}, v^{*}\right)$ :

$$
\begin{aligned}
& u^{*}=\frac{1}{2}\left[-\left(a+\frac{\delta}{\beta}-1\right)+\sqrt{\left(a+\frac{\delta}{\beta}-1\right)^{2}-4\left(\frac{\delta}{\beta}-a\right)}\right], \\
& v^{*}=\frac{\delta}{\beta}\left(u^{*}+b\right) .
\end{aligned}
$$

We assume that system (3) has only a positive equilibrium point $E\left(u^{*}, v^{*}\right)$ in the following, namely, $a-\frac{\delta}{\beta} b>0$. 
2.1 Stability analysis of the model without delay and diffusion

By linear stability analysis, the Jacobian matrix of (3) without diffusion at $E\left(u^{*}, v^{*}\right)$ is

$$
J=\left(\begin{array}{ll}
a_{11} & a_{12} \\
a_{21} & a_{22}
\end{array}\right)=\left(\begin{array}{rr}
1-2 u^{*}-\frac{a\left(1-u^{*}\right)}{u^{*}+a} & -\frac{u^{*}}{u^{*}+a} \\
\frac{\delta^{2}}{\beta} & -\delta
\end{array}\right) .
$$

Then, the corresponding characteristic equation is

$$
\lambda^{2}-\operatorname{tr} J \lambda+\operatorname{det} J=0,
$$

where

$$
\begin{aligned}
& \operatorname{tr} J=a_{11}+a_{22}=1-2 u^{*}-\frac{a\left(1-u^{*}\right)}{u^{*}+a}-\delta, \\
& \operatorname{det} J=a_{11} a_{22}-a_{12} a_{21}=\frac{\delta u^{*}}{u^{*}+a}\left(2 u^{*}+a+\frac{\delta}{\beta}-1\right) .
\end{aligned}
$$

The roots of Eq. (5) are

$$
\lambda_{1,2}=\frac{1}{2}\left(\operatorname{tr} J \pm \sqrt{(\operatorname{tr} J)^{2}-4 \operatorname{det} J}\right) .
$$

According to the Routh-Hurwitz Criterion, Eq. (5) has roots with negative real parts when $\operatorname{tr} J<0$ and $\operatorname{det} J>0$, namely, $\operatorname{Re} \lambda_{1,2}<0$ when $1-a-\delta>$ $0,(1-a-\delta)^{2}-8 \delta a<0$ and $a+\frac{\delta}{\beta}-1>0$. That is, the particular equilibrium point $E\left(u^{*}, v^{*}\right)$ is stable if

$$
\left\{\begin{array}{l}
1-a-\delta>0 \\
(1-a-\delta)^{2}-8 \delta a<0 \\
a+\frac{\delta}{\beta}-1>0 .
\end{array}\right.
$$

Lemma 1 Supposing that condition (7) holds, for system (3) without diffusion, $E\left(u^{*}, v^{*}\right)$ is asymptotically stable [9].

In the following, we assume that $\operatorname{tr} J<0$ and $\operatorname{det} J>0$. Namely, condition (7) is satisfied.

2.2 Stability analysis of the network diffusion model without delay

For system (3), the eigenvalues $\Lambda_{\alpha}$ and their corresponding eigenvectors $\phi^{(\alpha)}=$ $\left(\phi_{1}^{(\alpha)}, \ldots, \phi_{N}^{(\alpha)}\right)^{T}$ of network Laplacian matrix $L$ satisfy $\sum_{j=1}^{N} L_{i j} \phi_{j}^{(\alpha)}=\Lambda_{\alpha} \phi_{i}^{(\alpha)}$, where $\alpha=1, \ldots, N$, and $\Lambda_{\alpha} \leq 0$ because $L$ is a negative semidefinite real matrix. We assume that $\left|\Lambda_{\alpha}\right|$ is in decreasing order, namely, $\Lambda_{1} \leq \Lambda_{2} \leq \cdots \leq$ $\Lambda_{N}=0$.

Suppose Eq. (3) has the general solution of the following form:

$$
\left\{\begin{array}{l}
u_{i}=\sum_{\alpha=1}^{N} C_{\alpha}^{1} e^{\lambda_{\alpha} t} \phi_{i}^{(\alpha)} \\
v_{i}=\sum_{\alpha=1}^{N} C_{\alpha}^{2} e^{\lambda_{\alpha} t} \phi_{i}^{(\alpha)}
\end{array}\right.
$$


Substituting Eq. (8) into Eq. (3) give the following

$$
\lambda_{\alpha}\left(\begin{array}{c}
C_{\alpha}^{1} \\
C_{\alpha}^{2}
\end{array}\right)=\left(\begin{array}{cc}
a_{11}+d_{1} \Lambda_{\alpha} & a_{12} \\
a_{21} & a_{22}+d_{2} \Lambda_{\alpha}
\end{array}\right)\left(\begin{array}{c}
C_{\alpha}^{1} \\
C_{\alpha}^{2}
\end{array}\right)
$$

Thus, the characteristic equation of system (3) is

$$
\lambda_{\alpha}^{2}-p_{1} \lambda_{\alpha}+q_{1}=0
$$

where

$$
\begin{aligned}
& p_{1}=\operatorname{tr} J+\left(d_{1}+d_{2}\right) \Lambda_{\alpha} \\
& q_{1}=d_{1} d_{2} \Lambda_{\alpha}^{2}+\left(d_{1} a_{22}+d_{2} a_{11}\right) \Lambda_{\alpha}+\operatorname{det} J .
\end{aligned}
$$

The roots of Eq. (10) are $\lambda_{\alpha}=\frac{p_{1} \pm \sqrt{p_{1}^{2}-4 q_{1}}}{2}$. Then, for Eq. (10), the real part of the roots is negative when $p_{1}<0$ and $q_{1}>0$.

Theorem 1 Supposing that condition (7) holds, then for system (3), E( $\left.u^{*}, v^{*}\right)$ is asymptotically stable if the diffusion coefficients $d_{1}$ and $d_{2}$ satisfy

$$
s_{2} d_{1}<d_{2}<s_{1} d_{1}
$$

where $s_{1}=s_{+}, s_{2}=\max \left\{1, s_{-}, s_{3}\right\}, s_{ \pm}=\frac{2|J|-a_{11} a_{22} \pm 2 \sqrt{\operatorname{det} J\left(\operatorname{det} J-a_{11} a_{22}\right)}}{a_{11}^{2}}$, $s_{3}=-\frac{a_{22}}{a_{11}}$.

Proof Define

$$
q(z)=d_{1} d_{2} z^{2}+\left(d_{1} a_{22}+d_{2} a_{11}\right) z+\operatorname{det} J
$$

$z<0$. If $\triangle=\left(d_{1} a_{22}+d_{2} a_{11}\right)^{2}-4 d_{1} d_{2}$ det $J<0$ and $d_{1} a_{22}+d_{2} a_{11}>0$, namely, if $s_{2} d_{1}<d_{2}<s_{1} d_{1}, q(z)>0$ always holds, then $q_{1}>0$. In addition, it is evident that $p_{1}<0$ (due to that $\Lambda_{\alpha} \leq 0$ always holds). The proof is completed.

Remark. According to biological significance, $a_{11}>0$ must be held [16]. Therefore, $a_{11}>0$ if $\delta>\frac{2 a}{1+2 b-a} \beta$. Therefore, from $a_{11}+a_{22}<0$ and $d_{1} a_{22}+$ $d_{2} a_{11}>0$, we can calculate that the diffusion coefficients satisfy $d_{2}>d_{1}$ for system (3).

\section{Stability analysis of the predator-prey diffusion network with delay}

For system (2), the equilibrium point is clearly $E\left(u^{*}, v^{*}\right)$. Here, we use two methods $[29,30]$ to study the bifurcation dynamics property of system (2). 
3.1 Method I

In this section, according to the method in [29] and regarding the delay $\tau$ as the bifurcation parameter, we analyze the local stability of $E\left(u^{*}, v^{*}\right)$ and Hopf bifurcation.

By linear stability analysis and substituting Eq. (8) into Eq. (2), we can obtain

$$
\lambda_{\alpha}\left(\begin{array}{l}
C_{\alpha}^{1} \\
C_{\alpha}^{2}
\end{array}\right)=\left(\begin{array}{cc}
a_{11}+d_{1} \Lambda_{\alpha} & a_{12} e^{-\lambda \tau} \\
a_{21} & a_{22} e^{-\lambda \tau}+d_{2} \Lambda_{\alpha}
\end{array}\right)\left(\begin{array}{c}
C_{\alpha}^{1} \\
C_{\alpha}^{2}
\end{array}\right) .
$$

Therefore, the corresponding characteristic equation is

$$
\lambda^{2}-A_{\alpha} \lambda+B_{\alpha}+\left(C_{\alpha}-a_{22} \lambda\right) e^{-\lambda \tau}=0,
$$

where

$$
\begin{aligned}
& A_{\alpha}=a_{11}+\left(d_{1}+d_{2}\right) \Lambda_{\alpha}, \\
& B_{\alpha}=d_{1} d_{2} \Lambda_{\alpha}{ }^{2}+d_{2} a_{11} \Lambda_{\alpha}, \\
& C_{\alpha}=\operatorname{det} J+d_{1} a_{22} \Lambda_{\alpha} .
\end{aligned}
$$

When $\tau=0$, Eq. (12) becomes (10).

Lemma 2 Suppose (7) and (11) hold and regardless of what the network connection probability $p$ is, then Equation (12) has at least a simple pair of complex roots with zero real part $\pm i \omega_{\alpha+}$ when $\tau=\tau_{j}^{\alpha}$, where

$$
\begin{aligned}
& \omega_{\alpha+}=\sqrt{\frac{-\left(A_{\alpha}^{2}-a_{22}^{2}-2 B_{\alpha}\right)+\sqrt{\left(A_{\alpha}^{2}-a_{22}^{2}-2 B_{\alpha}\right)^{2}-4\left(B_{\alpha}^{2}-C_{\alpha}^{2}\right)}}{2}}, \\
& \tau_{j}^{\alpha}=\frac{1}{\omega_{\alpha+}} \arccos \left[\frac{\left(\omega_{\alpha+}^{2}-B_{\alpha}\right) C_{\alpha}-A_{\alpha} a_{22} \omega_{\alpha+}^{2}}{\omega_{\alpha+}^{2} a_{22}^{2}+C_{\alpha}^{2}}\right]+\frac{2 j \pi}{\omega_{\alpha+}}, j=0,1, \ldots
\end{aligned}
$$

Proof Suppose $i \omega$ is the root of (12), then

$$
-\omega^{2}-i \omega A_{\alpha}+B_{\alpha}+\left(C_{\alpha}-i \omega a_{22}\right)(\cos \omega \tau-i \sin \omega \tau)=0 .
$$

Separating the imaginary and real parts, we obtain

$$
\begin{aligned}
& \left(\omega^{2} a_{22}^{2}+C_{\alpha}^{2}\right) \sin \omega \tau=\left(-\omega^{2}+B_{\alpha}\right) \omega a_{22}-A_{\alpha} C_{\alpha} \omega, \\
& -\left(\omega^{2} a_{22}^{2}+C_{\alpha}^{2}\right) \cos \omega \tau=\left(-\omega^{2}+B_{\alpha}\right) C_{\alpha}+A_{\alpha} \omega^{2} a_{22} .
\end{aligned}
$$

From (14), we obtain

$$
\omega^{4}+\left(A_{\alpha}^{2}-a_{22}^{2}-2 B_{\alpha}\right) \omega^{2}+B_{\alpha}^{2}-C_{\alpha}^{2}=0 .
$$

The two roots of Eq. (15) can be expressed as follows:

$$
\omega_{\alpha \pm}^{2}=\frac{1}{2}\left[-\left(A_{\alpha}^{2}-a_{22}^{2}-2 B_{\alpha}\right) \pm \sqrt{\left(A_{\alpha}^{2}-a_{22}^{2}-2 B_{\alpha}\right)^{2}-4\left(B_{\alpha}^{2}-C_{\alpha}^{2}\right)}\right] .
$$

When conditions (7) and (11) hold, we have

$$
B_{\alpha}+C_{\alpha}=d_{1} d_{2} \Lambda_{\alpha}^{2}+\left(d_{1} a_{22}+d_{2} a_{11}\right) \Lambda_{\alpha}+\operatorname{det} J=q_{1}>0
$$

and

$$
B_{\alpha}-C_{\alpha}=d_{1} d_{2} \Lambda_{\alpha}^{2}+\left(-d_{1} a_{22}+d_{2} a_{11}\right) \Lambda_{\alpha}-\operatorname{det} J
$$


According to det $J>0, B_{\alpha}-C_{\alpha}=0$ has a negative root $\bar{\Lambda}_{\alpha}$, where $\bar{\Lambda}_{\alpha}=$ $\frac{-\left(d_{2} a_{11}-d_{1} a_{22}\right)-\sqrt{\left(d_{2} a_{11}-d_{1} a_{22}\right)^{2}+4 d_{1} d_{2} \text { detJ }}}{2 d_{1} d_{2}}$. That is $B_{\alpha}-C_{\alpha}>0$ when $\Lambda_{\alpha} \in$ $\left(-\infty, \bar{\Lambda}_{\alpha}\right)$ and $B_{\alpha}-C_{\alpha}<0$ when $\Lambda_{\alpha} \in\left(\bar{\Lambda}_{\alpha}, 0\right]$. Then, $B_{\alpha}^{2}-C_{\alpha}^{2}>0$ for $\Lambda_{\alpha} \in$ $\left(-\infty, \bar{\Lambda}_{\alpha}\right)$ and $B_{\alpha}^{2}-C_{\alpha}^{2}<0$ for $\Lambda_{\alpha} \in\left(\bar{\Lambda}_{\alpha}, 0\right]$. Hence, Eq. (15) has a positive root $\omega_{\alpha+}^{2}$ when $\Lambda_{\alpha} \in\left(\bar{\Lambda}_{\alpha}, 0\right]$. In addition, from $\Lambda_{1} \leq \Lambda_{2} \leq \cdots \leq \Lambda_{N}=0$, we know that $\Lambda_{\alpha} \leq 0$ always holds regardless of what $p$ is, so Equation (12) has at least one pair of roots with zero real part $\pm i \omega_{\alpha+}$ when $\tau=\tau_{j}^{\alpha}$, and $\tau_{j}^{\alpha}$ values are revealed from (14).

We denote $\lambda_{\alpha}(\tau)=\nu_{\alpha}(\tau)+i \omega_{\alpha}(\tau)$ as the root of Equation (12). Obviously, $\nu_{\alpha}\left(\tau_{j}^{\alpha}\right)=0$ and $\omega_{\alpha}\left(\tau_{j}^{\alpha}\right)=\omega_{\alpha+}$. In addition, we define $\tau^{*}=\min \left\{\tau_{j}^{\alpha}\right\}$. Then, we have the following theorem:

Theorem 2 Suppose (7) and (11) hold. For system (2), regardless of what $p$ is,

(i) if $\tau \in\left[0, \tau^{*}\right)$, then Eq. (12) has roots with a negative real part. Thus, the equilibrium $E\left(u^{*}, v^{*}\right)$ is asymptotically stable.

(ii) if $\tau>\tau^{*}$, then Eq. (12) has at least one root with a positive real part. Thus, the equilibrium $E\left(u^{*}, v^{*}\right)$ is unstable.

(iii) if $\tau=\tau^{*}$, then Eq. (12) has at least a pair of roots with zero real part $\pm i \omega_{\alpha+}$. In addition, $\left.\frac{d R e \lambda}{d \tau}\right|_{\tau=\tau^{*}}>0$. Thus, system (2) exhibits Hopf bifurcation at the equilibrium $E\left(u^{*}, v^{*}\right)$.

Proof From Eq. (12), we have

$$
\left(\frac{d \lambda}{d \tau}\right)^{-1}=\frac{\left(2 \lambda-A_{\alpha}\right) e^{\lambda \tau}-\left(C_{\alpha}-\lambda a_{22}\right) \tau-a_{22}}{\lambda\left(C_{\alpha}-\lambda a_{22}\right)} .
$$

Therefore, when conditions (7) and (11) hold and existing $\Lambda_{\alpha} \in\left(\bar{\Lambda}_{\alpha}, 0\right]$,

$$
\begin{aligned}
{\left[\left(\frac{d R e \lambda}{d \tau}\right)^{-1}\right]_{\tau=\tau^{*}} } & =\left[\operatorname{Re}\left(\frac{\left(-A_{\alpha}+2 i \omega_{\alpha+}\right) e^{i \omega_{\alpha+} \tau^{*}}+\left(C_{\alpha}-i a_{22} \omega_{\alpha+}\right) \tau^{*}-a_{22}}{\omega_{\alpha+}\left(a_{22} \omega_{\alpha+}+i C_{\alpha}\right)}\right)\right] \\
& =\frac{1}{a_{22}^{2} \omega_{\alpha+}^{2}+C_{\alpha}^{2}} \sqrt{\left(A_{\alpha}^{2}-a_{22}^{2}-2 B_{\alpha}\right)^{2}-4\left(B_{\alpha}^{2}-C_{\alpha}^{2}\right)}>0
\end{aligned}
$$

Therefore, from $\nu_{\alpha}\left(\tau^{*}\right)=0,\left.\frac{d \nu_{\alpha}}{d \tau}\right|_{\tau=\tau^{*}}>0$ and Lemma 2, we obtain $\tau^{*}$ as the bifurcation value. Furthermore, $\operatorname{Re} \lambda<0$ when $\tau<\tau^{*}, \operatorname{Re} \lambda<0$ is violated when $\tau>\tau^{*}$, and $\operatorname{Re} \lambda=0$ when $\tau=\tau^{*}$. Therefore, the proof is complete.

\subsection{Method II}

In this section, according to the method in [30] and assuming the delay $\tau$ to be small, we consider the Turing instability of system (2).

Because $\tau$ is small enough, we replace $v_{i}(t-\tau)=v_{i}(t)-\tau \frac{d v_{i}}{d t}$, and then, system (2) can be written as

$$
\left\{\begin{array}{l}
\frac{d u_{i}}{d t}=f\left(u_{i}, v_{i}-\tau \frac{d v_{i}}{d t}\right)+d_{1} \sum_{j=1}^{N} L_{i j} u_{j} \\
\frac{d v_{i}}{d t}=v_{i} h\left(u_{i}, v_{i}-\tau \frac{d v_{i}}{d t}\right)+d_{2} \sum_{j=1}^{N} L_{i j} v_{j}
\end{array}\right.
$$


where $g\left(u_{i}, v_{i}\right)=v_{i} h\left(u_{i}, v_{i}\right)$, and $g_{v_{i}}=\delta+2 v_{i} h_{v_{i}}$.

Expanding (16) in the Taylor series and neglecting the higher order nonlinearities, we have

$$
\left\{\begin{array}{l}
\frac{d u_{i}}{d t}=f\left(u_{i}, v_{i}\right)-\tau f_{v_{i}} \frac{d v_{i}}{d t}+d_{1} \sum_{j=1}^{N} L_{i j} u_{j}, \\
\frac{d v_{i}}{d t}=g\left(u_{i}, v_{i}\right)-\tau \frac{g_{v_{i}}-\delta}{2} \frac{d v_{i}}{d t}+d_{2} \sum_{j=1}^{N} L_{i j} v_{j} .
\end{array}\right.
$$

According to the research method in Section 2.2, we obtain the corresponding characteristic equation of the system at $E\left(u^{*}, v^{*}\right)$ as

$$
\lambda_{\alpha}^{2}-p_{2} \lambda_{\alpha}+q_{2}=0,
$$

where

$$
\begin{aligned}
& p_{2}=\frac{1}{1+\tau a_{22}}\left(\operatorname{tr} J+\left(d_{1}+d_{2}\right) \Lambda_{\alpha}+\tau\left(\operatorname{det} J+d_{1} a_{22} \Lambda_{\alpha}\right)\right), \\
& q_{2}=\frac{1}{1+\tau a_{22}}\left(d_{1} d_{2} \Lambda_{\alpha}^{2}+\left(d_{1} a_{22}+d_{2} a_{11}\right) \Lambda_{\alpha}+\operatorname{det} J\right) .
\end{aligned}
$$

Because $\tau$ is small, $\frac{1}{1+\tau a_{22}}>0$, namely, $\tau<\tau_{1}=-\frac{1}{a_{22}}$.

Turing instability means that system (2) without diffusion is stable (namely, when (7) holds), but unstable for diffusion system (2). According to the Routh-Hurwitz criterion, system (2) is stable when $p_{2}<0$ and $q_{2}>0$ always hold. When at least one of $p_{2}<0$ and $q_{2}>0$ is violated, Turing instability occurs. Therefore, we have the following theoretical results.

Theorem 3 Supposing $\tau<\tau_{1}$ and condition (7) hold, for system (2)

(i) If $\tau<\min \left\{\tau_{1}, \tau_{2}\right\}\left(\tau_{2}=-\frac{\text { trJ }}{\text { det.J }}\right)$ and $s_{2} d_{1}<d_{2}<s_{1} d_{1}$, then $p_{2}<0$ and $q_{2}>0$ hold. Thus, the equilibrium point $E\left(u^{*}, v^{*}\right)$ is asymptotically stable.

(ii) If $\tau<\min \left\{\tau_{1}, \tau_{2}\right\}, d_{2}>s_{1} d_{1}$ and existing $\Lambda_{\alpha} \in\left[\Lambda_{\alpha-}, \Lambda_{\alpha+}\right]$ for some $p$, then $q_{2}>0$ is violated. Thus, Turing bifurcation occurs at $E\left(u^{*}, v^{*}\right)$, where $\Lambda_{\alpha \pm}=\frac{-\left(d_{1} a_{22}+d_{2} a_{11}\right) \pm \sqrt{\triangle}}{2 d_{1} d_{2}}$.

(iii) If $\tau_{2}<\tau<\tau_{1}, s_{2} d_{1}<d_{2}<s_{1} d_{1}$ and existing $\Lambda_{\alpha}>\Lambda_{\alpha c}=-\frac{\text { trJ } J+\tau \operatorname{det} J}{d_{1}+d_{2}+d_{1} a_{22} \tau}$, then $p_{2}>0$ is violated. Thus, system (2) exhibits Turing bifurcation at $E\left(u^{*}, v^{*}\right)$.

Proof From $p_{2}=\frac{1}{1+\tau a_{22}}\left(\operatorname{tr} J+\left(d_{1}+d_{2}\right) \Lambda_{\alpha}+\tau\left(\operatorname{det} J+d_{1} a_{22} \Lambda_{\alpha}\right)\right)$, we know that $p_{2}<0$ when $\tau<\min \left\{\tau_{1}, \tau_{2}\right\}$. While $p_{2}>0$ when $\tau_{2}<\tau<\tau_{1}$ and existing $\Lambda_{\alpha}>\Lambda_{\alpha c}$. And from $q_{2}=\frac{1}{1+\tau a_{22}} q_{1}$, we know that $q_{2}>0$ if $\tau<\tau_{1}$ and $q_{1}>0$ hold. According to the proof of Theorem $1, q_{1}>0$ when $s_{2} d_{1}<$ $d_{2}<s_{1} d_{1} . q_{2}>0$ is violated when $\tau<\tau_{1}$, and some existing $\Lambda_{\alpha}$ satisfy $q_{1}<0$. By $q_{1}=d_{1} d_{2} \Lambda_{\alpha}^{2}+\left(d_{1} a_{22}+d_{2} a_{11}\right) \Lambda_{\alpha}+\operatorname{det} J$, we know that if $\Delta>0$, $d_{1} a_{22}+d_{2} a_{11}>0$ and existing $\Lambda_{\alpha} \in\left[\Lambda_{\alpha-}, \Lambda_{\alpha+}\right]$, namely when $d_{2}>s_{1} d_{1}$ and existing $\Lambda_{\alpha} \in\left[\Lambda_{\alpha-}, \Lambda_{\alpha+}\right]\left(\Lambda_{\alpha}\right.$ is related to $\left.p\right), q_{1}>0$ does not always hold. Therefore, we obtain conclusions (i), (ii) and (iii). The proof is complete.

\section{Numerical results}

In this section, based on the above theoretical results, we present some numerical simulations. Here, we construct a random network with $N=100$ nodes and consider the parameters as:

$$
a=0.08, b=0.04, \delta=0.6, \beta=0.6, d_{1}=0.02 .
$$


For this case, the positive equilibrium point is $E(0.1640,0.2040)$, and $\operatorname{tr} J=$ -0.201 , det $J=0.1645, s_{1}=6.8386$, and $s_{2}=1.5078$. These parameters satisfy condition (7). Therefore, according to Lemma 1, system (3) without diffusion is asymptotically stable [Figure 1]. If we choose $d_{1}=0.02$ and $d_{2}=0.1$, condition (11) holds. For system (3) and according to Theorem $1, E\left(u^{*}, v^{*}\right)$ is asymptotically stable, which is shown in Figure 2.

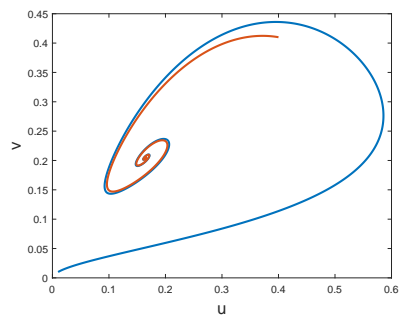

(a)

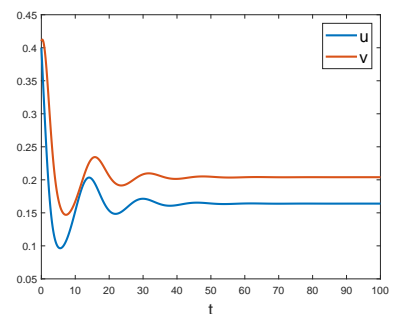

(b)

Fig. 1 When the parameters $a=0.08, b=0.04, \delta=0.6$, and $\beta=0.6$, (a)the phase space shows that system (2) without delay and diffusion has only one positive equilibrium point $E\left(u^{*}, v^{*}\right)$, (b)the time series diagram shows that $E\left(u^{*}, v^{*}\right)$ is stable.

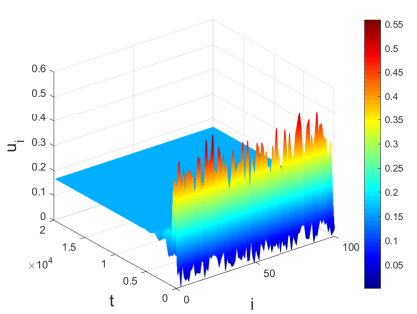

(a)

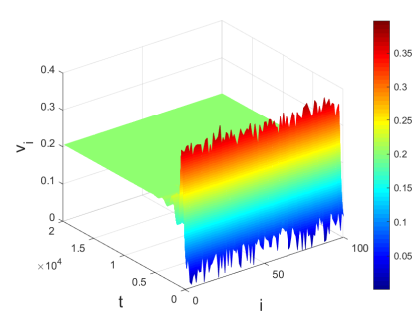

(b)

Fig. 2 When the parameters $a=0.08, b=0.04, \delta=0.6, \beta=0.6, d_{1}=0.02$, and $d_{2}=0.1$, the equilibrium point $E\left(u^{*}, v^{*}\right)$ of system (2) without delay is stable, where the initial values are randomly chosen and $p=0.1$.

When $\tau$ is small, we obtain $\tau_{1}=1.6667$ and $\tau_{2}=1.2285$. Considering $d_{2}$ and $\tau$ as the bifurcation parameters and from Theorem $3, E\left(u^{*}, v^{*}\right)$ is asymptotically stable when $\tau<\min \left\{\tau_{1}, \tau_{2}\right\}$ and $s_{2} d_{1}<d_{2}<s_{1} d_{1}$, which is shown in Figure 3 when $\tau=0.3657$ and $d_{2}=0.1$. In contrast, Turing instability occurs when $d_{2}>s_{1} d_{1}$ for existing $\Lambda_{\alpha} \in\left[\Lambda_{\alpha-}, \Lambda_{\alpha+}\right]$ or $\tau_{2}<\tau<\tau_{1}$ for existing $\Lambda_{\alpha}>\Lambda_{\alpha c}$. Therefore, we have two types of Turing instability. Figures 7 and 8 show that Turing bifurcation occurs when $p$ approximately satisfies $\frac{1}{N^{2}} \leq p \leq s_{1} \frac{\ln N}{N}$ (due to $\Lambda_{\alpha-}=-17.7705$ and $\Lambda_{\alpha+}=-0.9257$ ), where $\tau=0.5$ and $d_{2}=0.5$. Figure 9 shows the distribution of network eigenvalues $\Lambda_{\alpha}$ when 


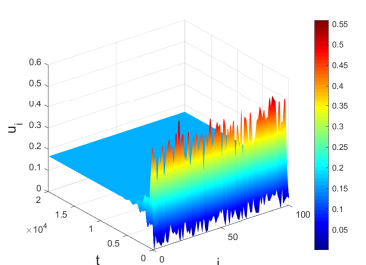

(a)

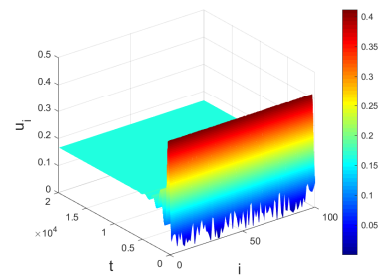

(c)

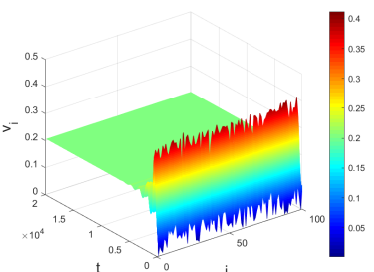

(b)

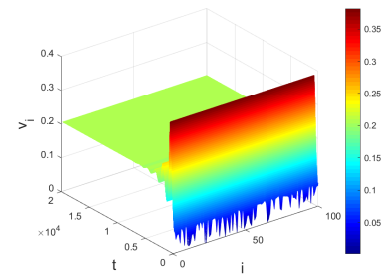

(d)

Fig. 3 For system (2), the equilibrium point $E\left(u^{*}, v^{*}\right)$ is asymptotically stable, where $\tau=$ $\tau^{*}-0.5, d_{1}=0.02$, and $d_{2}=0.1$, and the initial values are randomly chosen. (a) (b) $p=0.1$, (c) (d) $p=0.6$.

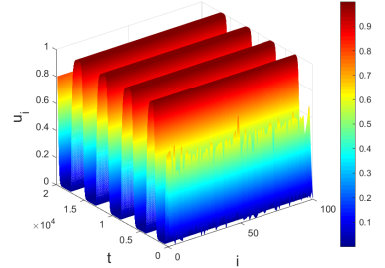

(a)

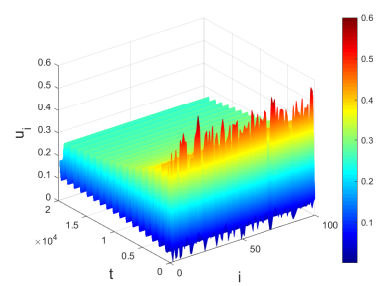

(c)

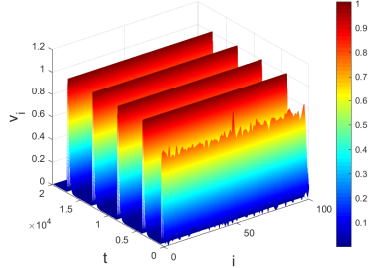

(b)

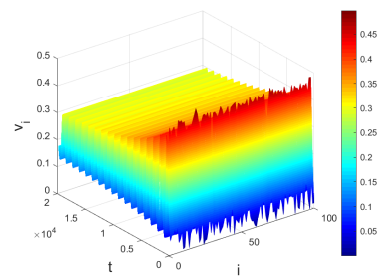

(d)

Fig. 4 For system (2), when $p=0.1, d_{1}=0.02, d_{2}=0.1$ and the initial values are randomly chosen, (a)(b)the equilibrium point $E\left(u^{*}, v^{*}\right)$ is unstable and there is a periodic solution when $\tau=\tau^{*}+1$, (c)(d)there is a Hopf bifurcation when $\tau=\tau^{*}$. 


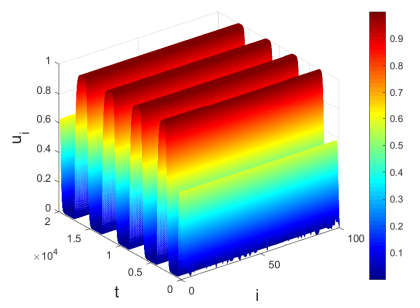

(a)

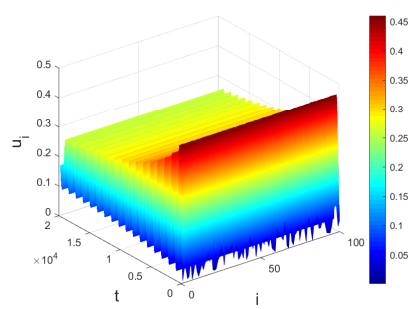

(c)

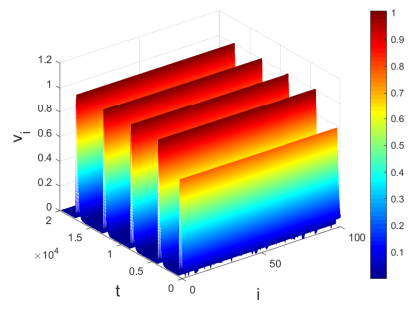

(b)

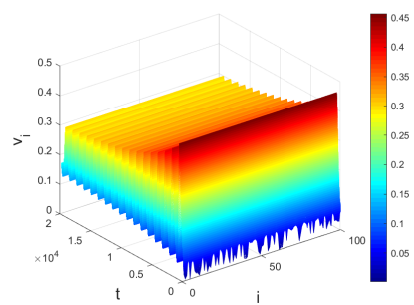

(d)

Fig. 5 For system (2), when $p=0.6, d_{1}=0.02, d_{2}=0.1$ and the initial values are randomly chosen, (a) (b)the equilibrium point $E\left(u^{*}, v^{*}\right)$ is unstable and there is a periodic solution when $\tau=\tau^{*}+1$, (c)(d)there is a Hopf bifurcation when $\tau=\tau^{*}$.

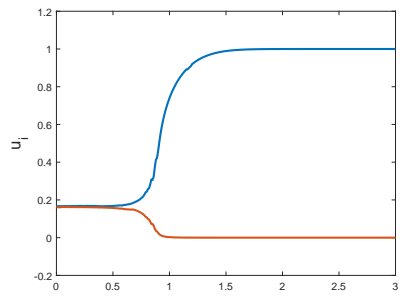

(a)

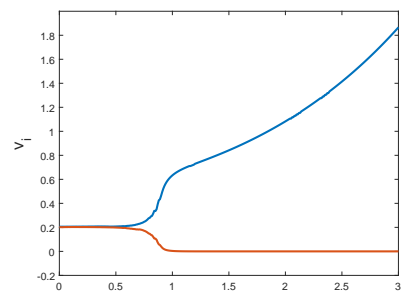

(b)

Fig. 6 Bifurcation diagram of system (2) with respect to $\tau$ when $d_{2}=0.1$.

$p=0.1 / 0.6$. Then, we give the approximate Turing bifurcation region of system (2) with respect to $d_{2}$ and $p$ [Figure 10]. Figure 11 shows the Turing bifurcation caused by delay and diffusion, where $\tau=1.5, d_{2}=0.1$, and $\Lambda_{\alpha c}=-0.4379$.

For system (2), taking $d_{2}=0.1$ and $p$ as randomly selected, we obtain that $\bar{\Lambda}_{\alpha}=-28.7563$ and $\tau^{*}=0.8657$. Therefore, considering delay $\tau$ as the bifurcation parameter and from Theorem $2, E\left(u^{*}, v^{*}\right)$ is asymptotically stable when $\tau \in\left[0, \tau^{*}\right)$, which is shown in Fig 3. When $\tau$ reaches the critical value $\tau^{*}$, the system undergoes Hopf bifurcation when $\tau=\tau^{*}$, and the system becomes unstable when $\tau>\tau^{*}$, where prey and predator will coexist and periodic oscillations will occur. The numerical simulations of the two cases are shown 


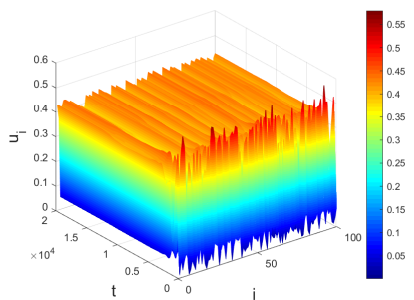

(a)

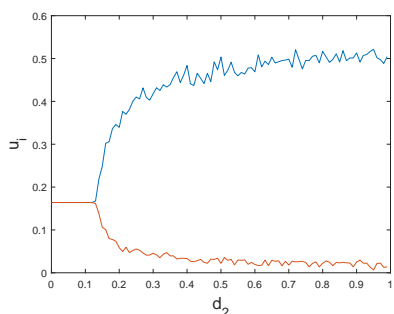

(c)

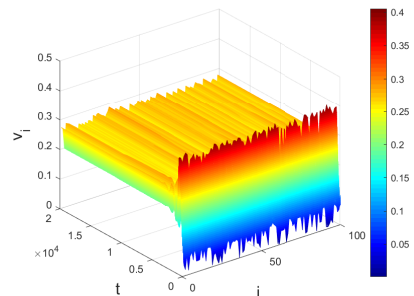

(b)

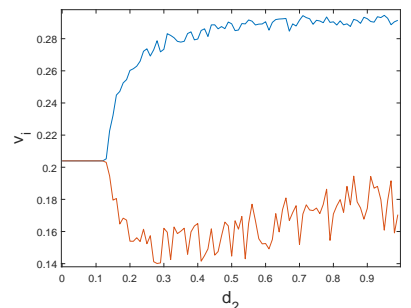

(d)

Fig. 7 When $d_{2}=0.5, \tau=0.5, p=0.1$ and the initial values are randomly chosen, system (2) is unstable and Turing bifurcation with respect to $d_{2}$ occurs.

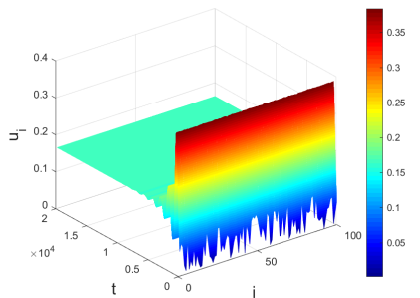

(a)

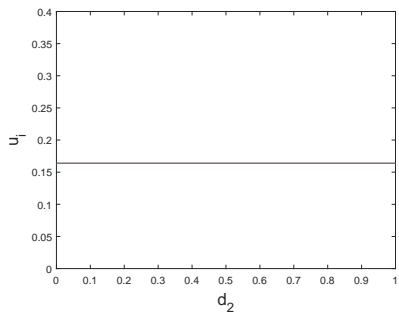

(c)

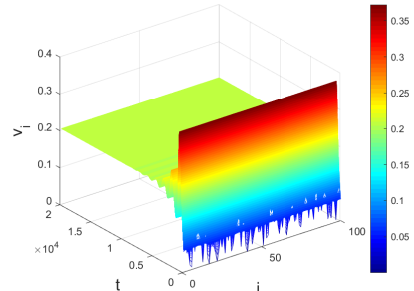

(b)

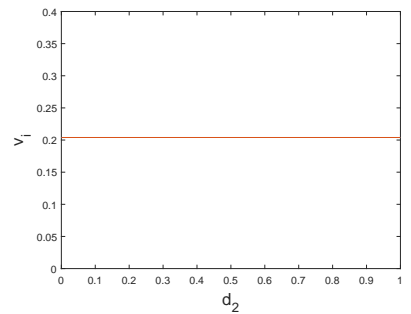

(d)

Fig. 8 When $d_{2}=0.5, \tau=0.5, p=0.6$ and the initial values are randomly chosen, there is no Turing bifurcation. Namely, system (2)is stable regardless of the $d_{2}$ value. 


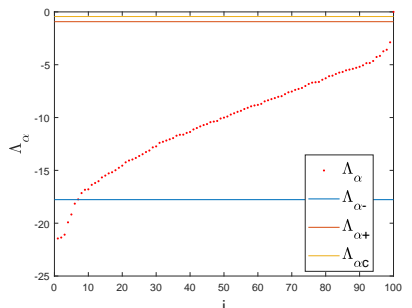

(a)

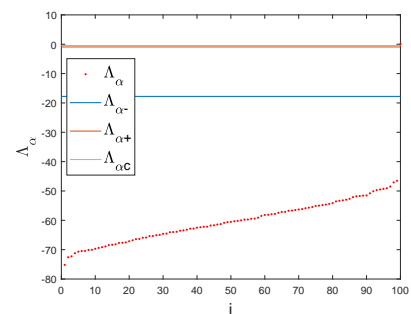

(b)

Fig. 9 The distribution of network eigenvalues $\Lambda_{\alpha}$. (a)There exist some $\Lambda_{\alpha}$ that satisfy $\Lambda_{\alpha} \in\left[\Lambda_{\alpha-}, \Lambda_{\alpha+}\right]$ and $\Lambda_{\alpha}>\Lambda_{\alpha c}$ when $p=0.1$. (b) $\Lambda_{\alpha} \in\left[\Lambda_{\alpha-}, \Lambda_{\alpha+}\right]$ is violated when $p=0.6$.

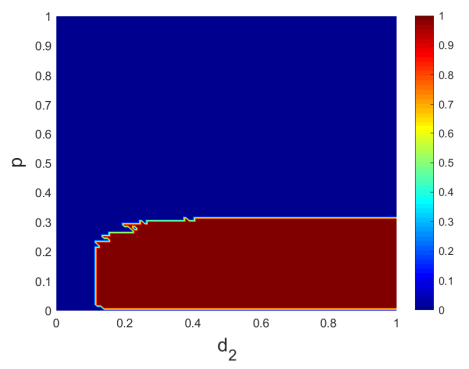

Fig. 10 Turing instability region of the network delay system (2) with respect to $p$ and $d_{2}$, where $\tau=0.5$ and the red region is the Turing instability region.

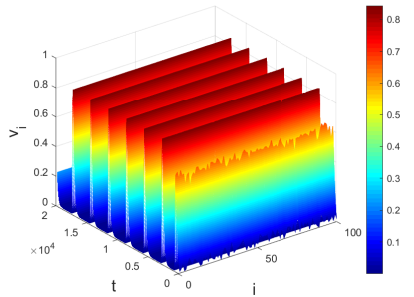

(a)

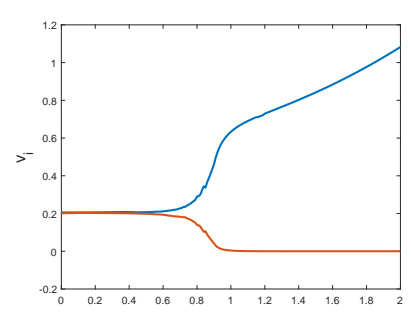

(b)

Fig. 11 When $d_{2}=0.1$ and $p=0.1$, (a)Turing instability occurs, where $\tau=1.5$ and the initial values are randomly chosen. (b)The corresponding Turing bifurcation graph with respect to $\tau$.

in Figures 4 and 5. Finally, we obtain the bifurcation diagram of $\tau$, which is shown in Figure 6. 


\section{Conclusion}

In this paper, a prey-predator diffusion network with delay is studied, and the stability of the positive equilibrium point $E\left(u^{*}, v^{*}\right)$ is investigated. From the linear stability analysis, we find that delay can change the stability and produce Hopf and Turing bifurcations. Then, the numerical simulations are given.

First, for the network model without delay, we give the conditions of asymptotic stability, and the corresponding figures are shown in Figures 1 and 2 . Then, for the network system with delay, we use two methods to analyze the influences of delay and diffusion on the stability (Theorems 2 and 3). Hopf and Turing bifurcations are obtained. When condition (11) holds, the network system always undergoes Hopf bifurcation and Turing bifurcation regardless of the network connection probability $p$ [Figures 3, 4, 5, 6, and 11]. Namely, regardless of how the network nodes are connected, prey and predators will coexist, and the system will oscillate periodically. If condition (11) is not held, Turing instability occurs when $p$ approximately satisfies $\frac{1}{N^{2}} \leq p \leq s_{1} \frac{\ln N}{N}$ $\left(\Lambda_{\alpha} \in\left[\Lambda_{\alpha-}, \Lambda_{\alpha+}\right]\right)$. Figures 7-10 show the numerical simulations. For this Turing instability caused by network diffusion, the network structure affects the stability. However, there are some problems that we have not studied, such as the stability and period as well as the direction of the bifurcating periodic solution. Thus, we will study these problems in the future.

\section{Acknowledgements}

This work is supported by the National Natural Science Foundation of China (11772291) and the Basic Research Project of Universities in Henan Province (21zx009).

\section{Conflict of interest}

The authors declare that there are no conflicts of interest.

\section{Data Availability Statements}

Data sharing not applicable to this article as no datasets were generated or analysed during the current study.

\section{References}

1. Turing, A.M.: The chemical basis of morphogenesis. Philos. Trans. R. Soc. Lond. Ser. B 237, 37-72 (1952)

2. Ouyang, Q., Swinney, H.L.: Transition from a uniform state to hexagonal and striped Turing patterns. Nature 352, 610-612 (1991) 
3. Zheng, Q.Q., Shen, J.W.: Turing instability in a gene network with cross-diffusion. Nonlinear Dyn. 78, 1301-1310(2014)

4. Zheng, Q.Q., Shen, J.W., Xu, Y.: Turing instability in the reaction-diffusion network. Phys. Rev. E 102, 062215 (2020)

5. Ji, Y.S., Shen, J.W.: Turing instability of Brusselator in the Reaction-Diffusion Network. Complexity 641, 1-12 (2020)

6. Tanner, J.T.: The stability and intrinsic growth rates of prey and predator populations. Ecology 56, 855-867 (1975)

7. Hsu, S.B., Hwang, T.W.: Global stability for a class of predator-prey systems. SIAM J. Appl. Math. 55, 763-783 (1995)

8. Ji, C.Y., Jiang, D.Q., Shi, N.Z.: Analysis of a predator-prey model with modified LeslieGower and Holling-type II schemes with stochastic perturbation. J. Math. Anal. Appl. 359, 482-498 (2009)

9. Aziz-Alaoui, M.A., Daher, O.M.: Boundedness and global stability for a predator-prey model with modiled Leslie-Gower and Holling type II shemes. App. Math. Lett. 16, 10691075 (2003)

10. Leslie, P.H.: Some further notes on the use of matrices in population mathematics. Biometrica 35, 213-245 (1948)

11. Peng, R., Wang, M.X., Positive steady states of the Holling-Tanner prey-predator model with diffusion. Proc. R. Soc. Edinb. A 135, 149-164 (2005)

12. Banerjee, M., Banerjee, S.: Turing instabilities and spatio-temporal chaos in ratiodependent Holling-Tanner model. Math. Bios. 236, 64-76 (2012)

13. Wang, W.M., Guo, Z.G., Upadhyay, R.K., Lin, Y.Z.: Pattern Formation in a CrossDiffusive Holling-Tanner Model. Discret. Dyn. Nat. Soc. 5560, 1-12 (2012)

14. Li, X., Jiang, W.H., Shi,J.P.: Hopf bifurcation and Turing instability in the reactiondiffusion Holling-Tanner predator-prey model. SIAM J. Appl. Math. 78, 287-306 (2013)

15. Zhou, J. Bifurcation analysis of a diffusive predatorCprey model with ratio-dependent Holling type III functional response. Nonlinear Dyn. 81, 1535-1552 (2015)

16. Camara, B.I., Aziz-Alaoui, M.A.: Turing and Hopf patterns formation in a predatorprey model with Leslie-Gower type functional response. Dynam. Cont. Discrete Ser. B 16 1-11 (2009)

17. Shi, H.B., Li, W.T., Guo, L.: Positive steady states of a diffusive predator-prey system with modified Holling-Tanner functional response. Nonlinear Anal. RWA 11, 3711-3721 (2010)

18. Othmer, H. G., Scriven, L. E.: Instability and dynamic pattern in cellular networks. J. theor. Biol. 32, 507-537 (1971)

19. Jansen, V.A.A., Lloyd,A.L.: Local stability analysis of spatially homogeneous solutions of multi-patch systems. J. Math. Biol. 41, 232-252 (2000)

20. Aly,S., Farkas, M.: Bifurcations in a predator-prey model in patchy environment with diffusion. Nonlinear Anal. RWA 5, 519-526 (2004)

21. Nakao,H., Mikhailov, A.S.: Turing patterns in network-organized activator-inhibitor systems. Nat.Phys. 6, 544-550 (2010)

22. Fernandes, L.D., de.Aguiar, M.A.M.: Turing patterns and apparent competition in predator-prey food webs on networks. Phys. Rev. E 86, 056203 (2012)

23. Kouvaris, N.E., Hata, S., Dìaz-Guilera, A.: Pattern formation in multiplex networks. Sci. Reports 5, 10840 (2015)

24. Zheng, Q.Q., Shen, J.W.: Turing instability induced by random network in fitzhughnagumo model. App. Math. Comput. 381125304 (2020)

25. Liu, C., Chang, L.L., Huang, Y., Wang, Z.: Turing patterns in a predator-prey model on complex networks. Nonlinear Dyn. 99, 3313-3322 (2020)

26. Nindjin, A.F., Aziz-Alaoui, M.A., Cadivel, M.: Analysis of a predator-prey model with modiled Leslie-Gower and Holling-Type II schemes with time delay. Nonlinear Anal. RWA 7, 1104-1118 (2006)

27. Shen, J.W., Liu, Z.R.,Zheng, W.X., Xu, F.D., Chen, L.N.: Oscillatory dynamics in a simple gene regulatory network mediated by small RNAs. Phys. A 388, 2995-3000 (2009)

28. Dong, T., Xu, W., Liao, X.F.: Hopf bifurcation analysis of reaction-diffusion neural oscillator system with excitatory-to-inhibitory connection and time delay. Nonlinear Dyn. 89, 2329-2345 (2017) 
29. Ruan,S.G.: Absolute stability, conditional stability and bifurcation in Kolmogorov-type predator-prey systems with discrete delays. Q. Appl. Math. 59, 159-173 (2001)

30. Sen, S., Ghosh, P., Riaz, S.S., Ray, D.S.: Time-delay-induced instabilities in reactiondiffusion systems. Phys. Rev. E 80, 046212 (2009)

31. Yang, R.Z., Zhang, C.R.: Dynamics in a diffusive predatorprey system with a constant prey refuge and delay. Nonlinear Anal. RWA 31, 1-22 (2016)

32. Chang, L.L., Liu, C., Sun,G.Q., Wang, Z., Jin, Z.: Delay induced patterns in a predatorprey model on complex networks with diffusion. New J. Phys. 21, 073035 (2019)

33. Wang, X.Y., Song, Z. Li, Z.Q., Chang, L.L., Wang, Z.: Delay-induced patterns in a reaction-diffusion system on complex networks. New J. Phys. 23, 073022 (2021) 